\title{
Site Diversity Technique Application on Rain Attenuation for Lagos
}

\author{
Abayomi Isiaka O. Yussuff ${ }^{1, \star}$, Nana Hamzat ${ }^{1}$, Nor Hisham Haji Khamis ${ }^{2}$ \\ ${ }^{1}$ Lagos State University, Lagos, Nigeria \\ ${ }^{2}$ Universiti Teknologi Malaysia, Skudai, Malaysia \\ e-mail: ayussuff@yahoo.com*, hisham@fke.utm.my
}

\begin{abstract}
This paper studied the impact of site diversity (SD) as a fade mitigation technique on rain attenuation at $12 \mathrm{GHz}$ for Lagos. SD is one of the most effective methods to overcome such large fades due to rain attenuation that takes advantage of the usually localized nature of intense rainfall by receiving the satellite downlink signal at two or more earth stations to minimize the prospect of potential diversity stations being simultaneously subjected to significant rain attenuation. One year (January to December 2011) hourly rain gauge data was sourced from the Nigerian Meteorological Agency (NIMET) for three sites (Ikeja, Ikorodu and Marina) in Lagos, Nigeria. Significant improvement in both performance and availability was observed with the application of SD technique; again, separation distance was seen to be responsible for this observed performance improvements.
\end{abstract}

Keywords: site diversity, rain rate, attenuation, slant path, FMT

\section{Introduction}

Satellite Communication requires the provisioning and deployment of two or more interconnected earth stations at spatially separated locations. Also, the necessity of operating at millimeter wavebands (Ku, Ka or Q/V bands) was informed by the eventual congestion of lower frequency bands resulting from commercial activities. It has been established that rain produces significant attenuation on radio waves of frequencies beyond $10 \mathrm{GHz}$. With respect to Ku band $(14 / 12 \mathrm{GHz}$. Diversity technique is one of the common fade mitigation techniques (FMTs) used in attempts to overcome signal fades resulting from convective rain events that typifies tropical stations. Frequency diversity (FD) employs the method of adaptively changing from frequency of propagation to a lower frequency to circumvent impending signal outages due to rain precipitations, and then switch back to the primary frequency after the disruptive events. Other diversity schemes include: Time diversity (TD), which is used to re-send the information at that time that propagation channel has been configured to allow it. Orbital diversity (OD), in which information is switched from one satellite to another. A major disadvantage of this technique is cost. Also, there is site diversity (SD), which is one of the most effective methods to overcome such large fades due to rain attenuation [1].

Site diversity is anchored on the proposition that the probability of attenuation being exceeded simultaneously at two sites is less than the probability of the same attenuation being exceeded at one of the sites by a factor which decreases with increasing distance between the sites and with increasing attenuation [2]. Intense rain cells cause large attenuation values on an earth-space link and often have horizontal dimensions of no more than a few kilometers. SD systems can re-route traffic to alternate earth stations with consequent considerable improvements in the system reliability. A balanced SD system (with attenuation thresholds on the two links equal) uses a prediction method that computes the joint probability of exceeding attenuation thresholds and is considered the most accurate and is preferred by ITU $[3,4]$. Site diversity takes advantage of the usually localized nature of intense rainfall by receiving the satellite downlink signal at two or more earth stations designed to minimize the prospect of potential diversity stations being simultaneously subjected to significant rain attenuation. Conceptually, a site diverse system comprises two or more spatially separated ground stations arranged in such a way to exploit the fact that the probability of attenuation due to rain will occur simultaneously on a typical slant path is significantly unlikely than the relative probability of attenuation occurrence on either individual paths. The effects of rain attenuation can then be 
consequently diminished or eliminated altogether. It has been suggested that the ground station with the higher received signal strength at any instant in time should be selected in order to significantly reduce the effect of rain attenuation [5].

Furthermore, rain has been identified as the major culprit for slant path signal impairment at frequencies above $10 \mathrm{GHz}$. It is also one of the most variable elements of weather. It varies in intensity, duration, frequency, and spatial pattern. The convective precipitations are of the highest rainfall intensities, localized, and also of short duration. While the medium or low rainfall intensities of stratiform precipitations have longer durations, and widespread. Rainfall intensity is an inverse function of its duration and can thus vary considerably with duration from one region to another, and for varying geographical features, such as the presence of mountains, hills or water masses, vegetation. The models predicting rain attenuation and those concerning the performance of site diversity systems can be The Hodge model [6], which is a regression model based on available attenuation statistics valid only for a few specific locations and Physical models based on the understanding of the rain process and the rainfall medium exhibiting a good performance globally [7-9]. The EXCELL [8], Matricciani [9] and Paraboni-Barbaliscia [10] models are well known physical prediction models for enhancement of site diversity performance. The Paraboni-Barbaliscia model [10] have been reported to submit that single and joint sites' rain attenuation indicate log-normal distributions, such that the site diversity gain can be derived from the measured single site rainfall rate and attenuation.

Ikeja with a geographical coordinates of $6.35 \mathrm{oN}$ (Lat.) and 3.23oE (Long.), Ikorodu $(6.600 \mathrm{~N}, 3.50 \mathrm{oE})$, and Marina $(6.45 \mathrm{oN}, 3.42 \mathrm{oE})$ are all stations located in Lagos, a coastal region located in the rain forest area in the southwestern tropical Nigeria. Lagos is bordered on the south by the Atlantic Ocean, and with mean annual rainfall of $1425 \mathrm{~mm}$ and altitude of $38 \mathrm{~m}$ above sea level.

\subsection{Site Diversity Gain}

Site diversity is one of the advanced techniques that employ a master and a remote station configuration separated several kilometres apart in order to take advantage of the inhomogeneity of convective rainfall, which occurs within localized rain cells with a diameter of a few tens of kilometres [11]. This has however been found to be much smaller than this [12] in tropical stations because of the peculiar nature of such regions. The inhomogeneity of rainfalls results in a decorrelation (depolarization) of the rain attenuation on the paths. Therefore, the prospect of transmitting and/ or receiving the signal via alternate paths reduces or eliminates the possibility of experiencing deep fades on both channels simultaneously. It has been observed that the deployment of multiple stations with the site separations in excess of the average horizontal dimensions of individual intense rain cells remarkably improved system availability because the joint path outages are presumed to be random and infrequent for diversity schemes [13]. Figures 1 and 2 depict the conceptual model and geometrical configuration of a typical two-terminal site diverse system. Hence, the jointly received signals are sent to the master station where they are further processed based on signal selection, switching, or a combination of both [8]. A diversity control unit coordinating the signal flow and a signal processing unit is incorporated at the master and the other earth stations respectively.

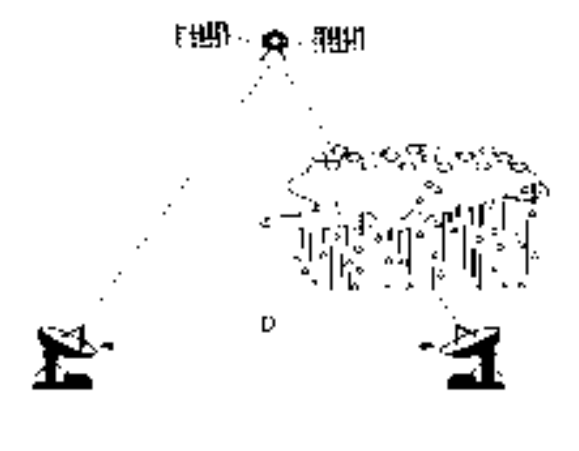

Figure 1. Conceptual model for site diversity

IJEEI Vol. 5, No. 1, March 2017 : 77 - 84 


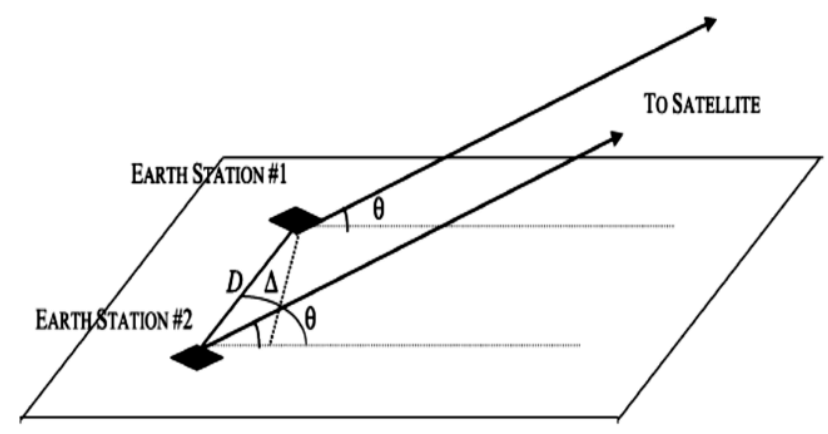

Figure 2. Geometrical configuration for 2-terminal diversity scheme 5[2]

\subsection{Models description}

Concerted efforts have been made over the past decade to develop reliable techniques for the prediction of path rain attenuation for a given location and frequency, and the availability of satellite beacon measurements has provided a database for the validation and refinement of the predictions models $[14,15]$. However, majority of these rain attenuation models were developed from data acquired from stations located in temperate regions [16]; with climatological characteristics far distinct from that experienced in tropical regions. Hence, the need for deliberate efforts to formulate rain attenuation prediction models specifically for the tropics. The ITU-R. Rec. P. 618-12 [3] is the globally accepted rain attenuation model for design and testing of any proposed model.

\subsubsection{Hodge model}

Hodge [6] proposed the first empirical prediction model for site diversity gain as a product function of Individual gains contributed by single site attenuation, site separation, baseline orientation, link frequency and path elevation angle. This model has been adopted by ITU-R. Rec. P. 618-12 [3] for the estimation of gain up to a site separation of $20 \mathrm{~km}$; and it is represented mathematically as:

$$
G_{S D}=G_{D} * G_{\theta} * G_{f} * G_{\Delta}
$$

In the above relationship, $G_{D}, G_{\theta}, G_{f}, G_{\Delta}, G_{D}$ are factors expressing the dependence of the site diversity gain on the the site separation distance, $\mathrm{d}(\mathrm{km})$, the common elevation angle of both slant paths, $\theta$ (degrees), the frequency of operation, $f(\mathrm{GHz})$, and the orientation of the baseline between the two earth stations, $\Delta$ (degrees). Each dependence factor is given by the expression:

$$
G_{D}=a\left(1-e^{b d}\right)
$$

where:

$$
a=0.78 A-1.94\left(1-e^{-0.11 A}\right), b=0.59\left(1-e^{-0.1 A}\right), G_{\theta}=1+0.006 \theta, G_{f}=\exp (-0.025 f),
$$

and $G_{\Delta}=1+0.002 \Delta$

Again, the diversity gain, $G_{S D}$ is the difference between the single site antenna attenuation and the joint diversity systems for a specified percentage of probability, $p \%$. Hodge's model is particularly suitable for Ku band although the diversity gain here is observed to be smaller than what is achievable on Ka band. Also, it has been noted that the diversity gain sharply reduces for elevation angles that are below 30 degrees. This is premised on the fact that for lower elevation angles, the slant path tends to be longer and hence, the higher probabilities of experiencing signal propagation impairments due to rainfall. This is especially true for stations located in tropical regions. However, for separation distances in excess of 20 
$\mathrm{km}$, the Paraboni-Barbaliscia model [10] has been co-opted into the ITU-R. Rec. P. 618-12 model [3].

\section{Research Method}

The slant path rain attenuation values were computed in line with the procedure outlined in ITU-R. Rec. P. 618-12 [3], The rain rate $(\mathrm{mm} / \mathrm{hr})$ recorded at time $t$ for each pixel location indicating the reference and diversity earth stations were used to compute the specific attenuation $\gamma_{0.01}(\mathrm{~dB} / \mathrm{km})$ given by Equation (4), where $a$ and $b$ are the link frequency dependent coefficients such as frequency, rain drop size, polarization, antenna's elevation angle and temperature, are defined in [3].

$$
\gamma_{t}=a R_{t}^{b}
$$

More so, ITU-R Rec. P. 618-12 [3] rain attenuation prediction model is used to predicts the long-term statistics of the slant-path rain attenuation at a given location for frequencies up to $55 \mathrm{GHz}$, rainfall rate at $0.01 \%$ of the time $\left(A_{0.01}\right)$ as the basic input and with subsequent resulting attenuation at $0.01 \%\left(A_{0.01}\right)$ applied as the basis for estimating the attenuation exceeded at other percentages $\left(A_{\%_{p}}\right)$. The step-by-step procedures for calculating rain attenuation cumulative distribution function over the satellite link are as follow:

The effective path length $L_{\text {eff }}(\mathrm{km})$ through rain is obtained by multiplying the horizontally adjusted slant path by the vertical reduction factor:

$$
L_{e f f}=L_{h 0.01} r_{v 0.01}(\mathrm{~km})
$$

Therefore predicted slant path attenuation exceeded for $0.01 \%$ of an average year is

$$
A_{0.01}=\gamma_{0.01} L_{e f f}(d B / k m)
$$

Hence, the predicted attenuation exceeded for other percentages $\% p$ of an average year may be obtained from the value of $A_{0.01}$ by using the following extrapolation according to ITU-R P. 618-12 [3]. That is:

$$
A_{\% p}=A_{0.01}\left(\frac{p}{0.01}\right)^{-\left[0.655+0.033 \ln p-0.045 \ln A_{0.01}-z \sin \theta(1-p)\right]}(d B)
$$

Where $p$ is the percentage probability of interest and $z$ is given by

$$
\begin{aligned}
& \text { For } p \geq 1.0 \%, z=0 \\
& \text { For } p<1.0 \%, z=\left\{\begin{array}{l}
0 ; \text { for } / \phi \mid \geq 36^{\circ} \\
z=-0.005(|\phi|-36) \text { for } \theta \geq 25^{\circ} \text { and }|\phi|<36^{\circ} \\
z=-0.005(\mid \phi-36 /)+1.8-4.25 \sin \theta, \text { for } \theta<25^{\circ} \text { and }|\phi|<36^{\circ}
\end{array}\right.
\end{aligned}
$$

\subsection{Data Collection}

One year (January to December 2011) hourly rain gauge data was sourced from the Nigerian Meteorological Agency (NIMET). Measurement setup comprise buck-type rain gauge installed at the measurement at the three sites (Ikeja, Ikorodu and Marina) to record the rain rate exceedances. One-minute rainfall rate was subsequently obtained from these data using the empirical model developed by Chebil and Rahman [17,18]. The 1-minute rain rate for arbitrary percentage of time, $p \%$; and $R_{p}$ can be estimated by using only the average annual total rainfall. The model is given by Equations (10) through (12).

IJEEI Vol. 5, No. 1, March 2017: $77-84$ 
Furthermore, the rain rate, $R(\mathrm{~mm} / \mathrm{h})$ were recorded for different \% probability, $p$ indicating the reference (Ikeja) and diverse earth stations (Marina and Ikorodu) are shown in Table 1 below. Chebil and Rahman's [15,16] proposed rain rate conversion model for converting these hourly data to the equivalent one-minute rainfall rate values was applied as follow:

$$
\begin{aligned}
& C F_{60}=\mathrm{R}_{1(\mathrm{P})} / \mathrm{R}_{60(\mathrm{P})} \\
& C F_{60}=0.772 * p^{-0.041}+1.141 * \exp (-2.57 * p)
\end{aligned}
$$

where $C F_{60}$ is the rain rate conversion factor, defined as the ratio of rain rates $R_{1}$ at a given percentage of time $p$ with an integration time of 1 and 60 minutes, respectively. The constraint of this model is: $0.001 \% \leq p \leq 1.0 \%$; hence $\mathrm{R}_{1(\mathrm{P})}$ the 1 -minute rain rate is:

$$
\mathrm{R}_{1(\mathrm{P})} /=\mathrm{R}_{60(\mathrm{P})} * C F 60
$$

Table 1. One-minute rain rate for selected locations

\begin{tabular}{ccccccccccc}
\hline PROBABILTY & 0.001 & 0.002 & 0.003 & 0.005 & 0.01 & 0.02 & 0.03 & 0.1 & 0.5 & 1.0 \\
\hline IKEJA $(\mathrm{mm} / \mathrm{h})$ & 119.8 & 118.0 & 116.9 & 115.5 & 113.2 & 110.2 & 107.8 & 95.8 & 61.5 & 47.6 \\
MARINA $(\mathrm{mm} / \mathrm{h})$ & 126.0 & 124.1 & 123.0 & 121.5 & 119.1 & 115.9 & 113.5 & 100.8 & 64.7 & 50.1 \\
IKORODU $(\mathrm{mm} / \mathrm{h})$ & 129.4 & 127.5 & 126.3 & 124.8 & 122.3 & 119.1 & 116.5 & 103.5 & 66.4 & 51.4 \\
\hline
\end{tabular}

Ikorodu and Marina stations were set at $16.67 \mathrm{~km}$ and $16.69 \mathrm{~km}$ apart, as shown in Figure 3. Figure 3 displays the images of the selected locations using Google Map Ruler to determine the site line-of-sight (LOS) separation distances between the reference station and the two other stations.

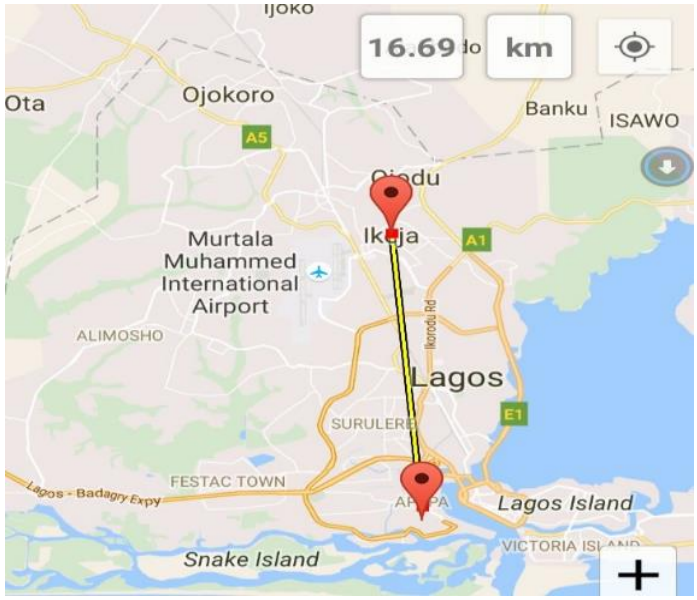

(a)

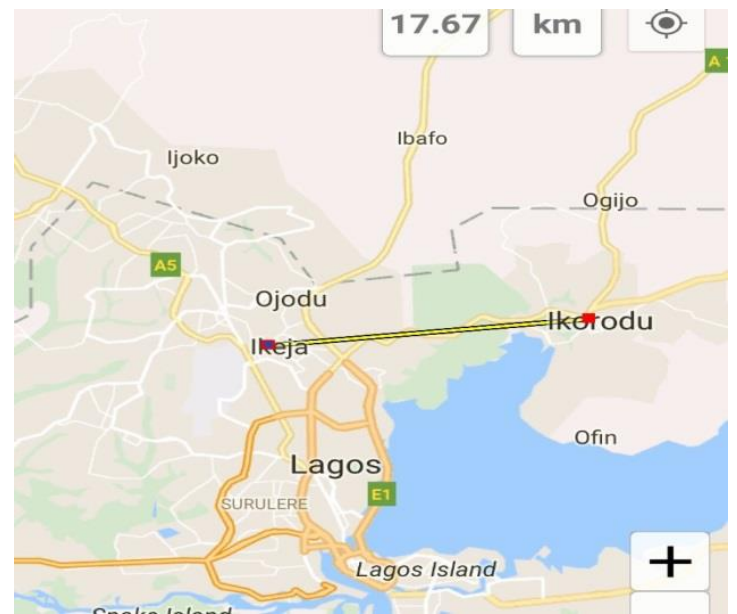

(b)

Figure 3. Site separation distances between (a) Ikeja and Marina, and (b) Ikeja and Ikorodu

In Table 2, the parameters used in determining the site diversity gains for the respective earth locations are shown.

Table 2. Climatological parameters for selected stations

\begin{tabular}{cc}
\hline Parameters & Values \\
\hline Elevation angle (degree) & 44.5 \\
Frequency (gigahertz) & 12 \\
Base line orientation (degree) & 45 \\
Site separation distance (km) & 16.69 \\
(IKEJA - MARINA) & \\
Site separation distance (km) & 17.67 \\
(IKEJA - IKORODU) & \\
\hline
\end{tabular}


Furthermore, the rain rate values at different percentage of time were plotted and analyzed using some programming codes in MATLAB. The diversity improvement factor, I which is the ratio of the probabilities for a specific attenuation exceeded, is subsequently derived. According to ITU-R. Rec. P.618-12 [3], the diversity improvement factor model is given as:

$$
\mathrm{I}=\mathrm{P} 1 / \mathrm{P} 2=1 /\left(1+\beta^{2}\right) *\left(1+\frac{100 \beta^{2}}{\mathrm{P} 1}\right) \approx 1+100 \beta^{2} / \mathrm{P} 1
$$

where $\beta^{2}=d^{1.33} * 10^{-4}$, $\mathrm{d}$ is the seation distance while P1 and P2 refer to the percentages of time for the observed single and joint's rain attenuation exceeded respectively.

\section{Results and Analysis}

Figure 4.0 shows the cumulative distribution function (CDF) of path attenuation and rain rates for Ikeja, Ikorodu and Marina, Lagos. The plot of the individual site's slant path attenuation exceeded against the corresponding attenuation gain are shown in Figure 5, while Figure 6 displays the evident improvement in performance in terms of reduction in rain attenuation effects accrued to implementing joint diversity system. It is clearly seen that there is significant reduction in attenuation exceeded in Figure 6.

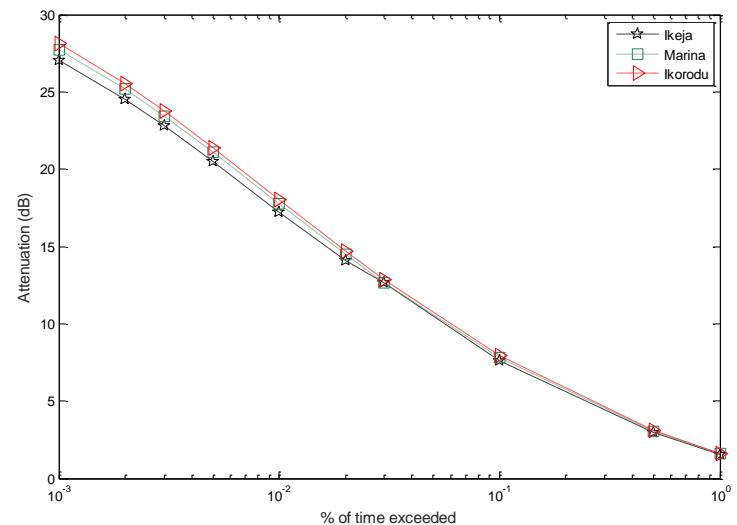

(a)

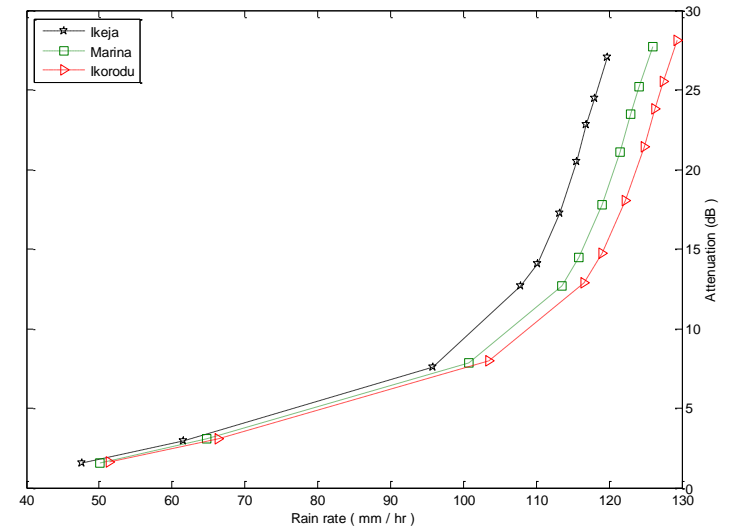

(b)

Figure 4. (a) CDFs of attenuation for and (b) Attenuation against. rain rate for Ikeja, Marina and and Ikorodu.

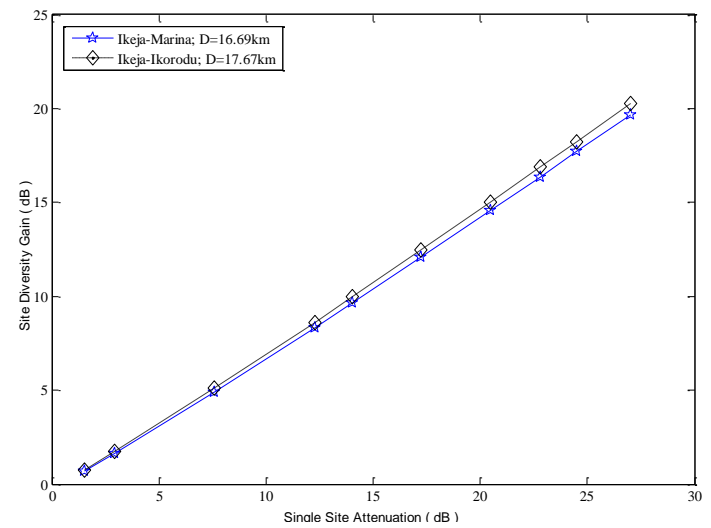

Figure 5. Site diversity gain vs. single site attenuation

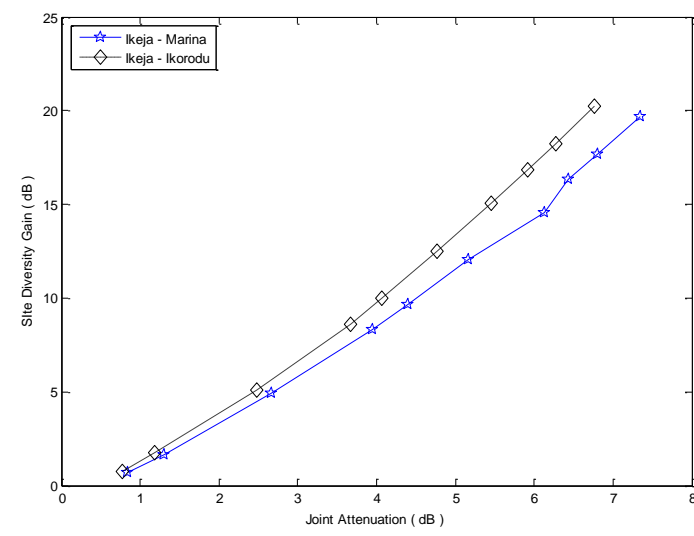

Figure 6. Site diversity gain versus joint attenuation 
Moreover, site separation distance is the major factor that affects the amount of diversity gain for any two diverse systems. Shown in Figure 7 is the plot of diversity gains $\left(G_{S D}\right.$, which consists of $G_{S D 1}$ and $G_{S D 2}$ ) improvement factors $I_{1}$ and $I_{2}$ ) and comparison of attenuation exceedances for single and joint diversity systems.

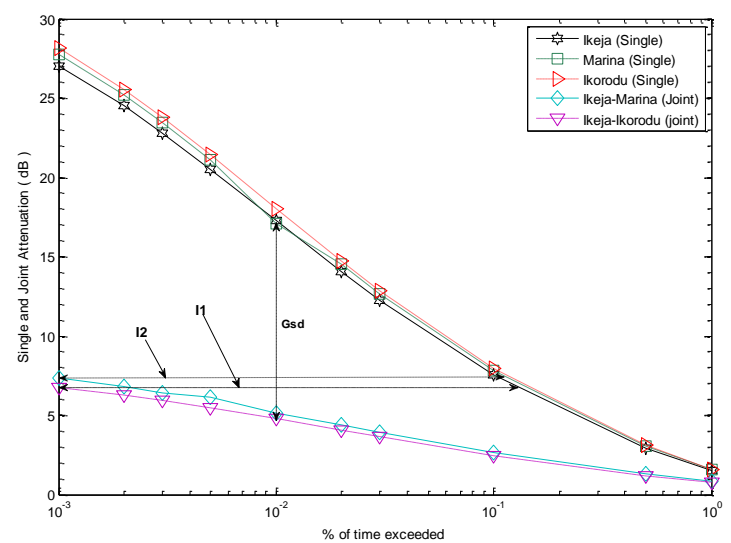

Figure 7. Comparison of single and joint attenuation

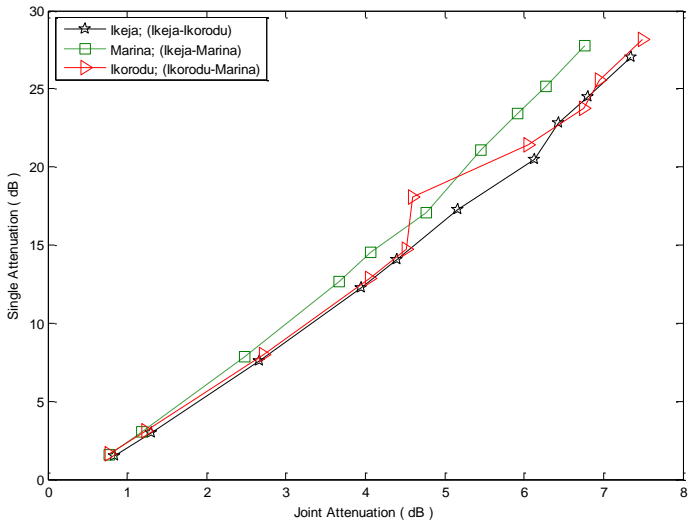

Figure 8. CDFs of single site attenuation site against joint attenuation

Here, $\mathrm{G}_{\mathrm{SD} 1}$ and $\mathrm{G}_{\mathrm{SD} 2}$ are found to be12.07 $\mathrm{dB}$ and $12.48 \mathrm{~dB}$ respectively, while $\mathrm{I}_{1} \approx \mathrm{I}_{2} \approx$ 43.23 .

In summary, the improved link availability for distances $16.69 \mathrm{~km}$ (Ikeja to Marina) and $17.67 \mathrm{~km}$ (Ikeja to Ikorodu) for $0.01 \%$ of the time exceeded are $99.9998 \%$ and $99.9999 \%$ respectively. Hence, site diversity provides significant improvement in both performance and availability of the system. Figure 8 depicts the effect of applying Hodge model to a separation distance exceeding the recommended maximum distance of $20 \mathrm{~km}$ (in this instance, $22.70 \mathrm{~km}$ ). It can be observed that there is no significant increment in joint attenuation between Ikeja to Ikorodu $(17.67 \mathrm{~km})$ and Ikorodu to Marina $(22.70 \mathrm{~km})$. Also, the Ikorodu-Marina system largely deviate from the Hodge model's trend between $p=0.02 \%$ and $p=0.003 \%$. This effect is more pronounced at $p=0.01 \%$ with a corresponding attenuation of $4.06 \mathrm{~dB}$.

\section{Conclusion}

This effect of site separation distance with keeping other factors such as propagation frequency, antenna elevation angle, polarization, and baseline orientation angle constant was investigated in this work. Significant improvement in both performance and availability of the site diversity system was observed; and it is safe to conclude that separation distance is a key factor to be considered when opting for site diversity as propagation impairment mitigation technique. Furthermore, efficiency starts to diminish and performance begins to suffer when the $20 \mathrm{~km}$ maximum separation distance threshold as recommended by ITU-R. Rec. P. 618-12 is exceeded.

\section{References}

[1] Ippolito LJ. Satellite Communications Systems Engineering: Atmospheric Effects. Satellite Link Design and System Performance. Hoboken, NJ: Wiley Publication. 2008.

[2] ITU-R BO. 791. Choice of Polarization for the Broadcasting-Satellite Service. Geneva. 2002.

[3] ITU-R. P. 618-12, Propagation Data and Prediction Methods Required for the Design of Earth-Space Telecommunications Systems, in Recommendation ITU-R P Series. 2015.

[4] Yussuff Al, Khamis NH. Rain Attenuation Modelling and Mitigation in the Tropics: Brief Review. International Journal of Electrical and Computer Engineering. 2012; 2(6): 748-757. 
[5] Athanasios D. et al. Long-Term Rain Attenuation Probability and Site Diversity Gain Prediction Formulas. IEEE Transactions on Antennas and Propagation. 2005; 53(7): 2307-2313.

[6] Hodge DB. An improved model for diversity gain in earth-space propagation paths. Radio Sci. 1982; 17(6): 1393-1399.

[7] Kanellopouloset JD. al. Rain attenuation problems affecting the performance of microwave communication systems. Ann. Telecommun. 1990; 45(7-8): 437-451.

[8] Bosisio AV, Riva C. A novel method for the statistical prediction of rain attenuation in site diversity systems: Theory and comparative testing against experimental data. Int. J. Satellite. Communication. 1998; 16: 47-52.

[9] Matricciani E. Prediction of site diversity performance in satellite communications systems affected by rain attenuation: Extension of the two layer rain model. European Trans. Telecommunication. 1994; 5(3): 27-36.

[10] Luglio M. et al. Large-scale site diversity for satellite communication networks. Int. J. Satellite Commun. 2002; 20: 251-260.

[11] Lin SH. Method for calculating rain attenuation distribution on microwave paths. Bell Syst. Tech. J. 1975; 54(6): 1051-1086.

[12] Khamis NH. H. Path Reduction Factor for Microwave Terrestrial Links Derived from the Malaysian Meteorological Radar Data. PhD Thesis. Universiti Teknologi Malaysia, Malaysia; 2005.

[13] Allnutt J. E. Satellite-to-Ground Radiowave Propagation. Second Edition. IET Electromagnetic wave series 54. 2011: 293.

[14] Tseng C. H. et al. Prediction of Ka-band terrestrial rain attenuation using 2-year rain drop size distribution measurements in Northern Taiwan. Journal of Electromagnetic Waves and Applications. 2005; 19(13): 1833-1841.

[15] Li Y, Yang P. The permittivity based on electromagnetic wave attenuation for rain medium and its applications. Journal of Electromagnetic Waves and Applications. 2006; 20(15): 2231-2238.

[16] Singh M. S. et al. Rain attenuation model for S. E. Asia countries. IET Electronic Letters. 2007; 43(2): 75-77.

[17] Chebil J, Rahman T.A. Rain rate statistical conversion for the prediction of rain attenuation in Malaysia. Electronic Letts. 1999; 35: 1019-1021.

[18] Chebil J, Rahman T.A. Development of 1-min rain rate contour map for microwave application in Malaysia Peninsula. Electronics Letts.1999; 35: 1712-1774.

IJEEI Vol. 5, No. 1, March 2017: $77-84$ 